\title{
Appropriate Use of Cardiac Magnetic Resonance Imaging and Its Impact on Downstream Resource Utilization
}

\author{
Tiberio Frisoli, Joseph Gibbs, Karthik Ananthasubramaniam \\ Heart and Vascular Institute, Henry Ford Hospital, Detroit, MI, USA \\ Email:kananth1@hfhs.org
}

How to cite this paper: Frisoli, T., Gibbs, J. and Ananthasubramaniam, K. (2017) Appropriate Use of Cardiac Magnetic Resonance Imaging and Its Impact on Downstream Resource Utilization. World Journal of Cardiovascular Diseases, 7, 243-250. https://doi.org/10.4236/wjcd.2017.78023

Received: July 10, 2017

Accepted: August 8, 2017

Published: August 11, 2017

Copyright $\odot 2017$ by authors and Scientific Research Publishing Inc. This work is licensed under the Creative Commons Attribution International License (CC BY 4.0).

http://creativecommons.org/licenses/by/4.0/

\begin{abstract}
Purpose: Echocardiography and nuclear perfusion imaging studies ordered for appropriate indications by Appropriate Use Criteria (AUC) have been shown more likely to impact management than studies deemed inappropriate or uncertain. We sought to evaluate the appropriateness of indications for all cardiac magnetic resonance imaging (CMR) testing done at our institution in 2011, and to what extent appropriateness of CMR was associated with impact on patient management. Methods: This was a single-center retrospective cohort study including 239 consecutive patients who received CMR over the 12-month calendar period in 2011. CMR studies were classified as appropriate, uncertain or inappropriate based on the 2006 AUC. A study was deemed to have had impact if it changed or clarified the patient's diagnosis or directly influenced downstream decision-making by subspecialty referral, further testing or treatment changes. Results: The most common indications for CMR included evaluation of cardiomyopathy (79\%), valvular pathology (8\%), suspected myocarditis (8\%), and cardiac masses (5\%). Of CMRs performed, $96 \%$ were appropriate, $1 \%$ inappropriate, and 3\% uncertain. Appropriate CMRs were more likely to be associated with changes in management $(90 \%)$ than inappropriate or uncertain studies (40\%), with an odds ratio of 21.5:1. The most common reasons CMR classified as appropriate did not change management were 1) incomplete study, 2) physician judgment not to take action based on the CMR result and 3) hypertrophic cardiomyopathy in specific circumstances. Conclusions: Appropriate CMR studies were more likely to impact management than inappropriate/uncertain studies. When ordered for appropriate indications, CMR has demonstrable impact on patient management decisions.
\end{abstract}

\section{Keywords}

Cardiac MRI, Appropriate Use Criteria, Utilization, Cardiac Imaging 


\section{Introduction}

Increased utilization of cardiac imaging has been implicated as a major contributor in the overall escalation in the cost of health care. Amongst Medicare recipients alone, annual expenditures have universally increased across physician fees, testing, and part B drugs, with the greatest increases observed in testing with medical imaging. This area alone demonstrated more rapid growth than any other billed service between 1999 and 2003. In fact, imaging modalities have demonstrated periods of annual growth as great as $20 \%$, as observed between 2001 and 2002 [1]. In response to these rapidly rising costs, increased scrutiny is being given to cardiac testing in an effort to ensure the appropriate use of imaging studies. Development of the 2006 Appropriate Use Criteria (AUC), and later its revision in 2013 have increasing implications regarding Centers for Medicare and Medicaid Services reimbursement. With approval of the Protecting Access to Medicare Act, physicians will soon be asked to provide certification that the AUC were consulted with test orders, and may ultimately be penalized if their ordering practices do not closely adhere to defined ordering standards [2] [3] [4].

Cardiac magnetic resonance (CMR) has likewise seen increasing use over recent years. Currently, the AUC for CMR describe 80 possible clinical application scenarios, characterized by expert opinion as "appropriate", "maybe appropriate", or "rarely appropriate" [5]. Limited data exist in which these studies are demonstrated to have impact on management or how they affect downstream utilization. This study was designed to evaluate the appropriateness of CMRs ordered at our institution in 2011 as well as its downstream impact on patient management, with specific regard to changes in therapy (i.e. medications added or subtracted, revascularization cancelled or modified, defibrillator placed, etc.) or changes in further downstream testing.

\section{Materials and Methods}

\subsection{Study Population}

Two hundred thirty nine consecutive patients referred for a clinically indicated CMR between January 1, 2011 and December 31, 2011 were retrospectively identified based on the completed order for CMR. All patients receiving CMR during the study period were included in the study cohort. Patient demographics and cardiac risk factors as known at the time of presentation for CMR were abstracted from the medical record by physician reviewers using a composite of most recent outpatient visit notes, inpatient admission documentation as well as other recorded medical history in the electronic medical record. Indication for CMR and the result of the study were also obtained. Downstream testing, medication changes, as well as interventions performed during the 90 days following CMR were likewise evaluated. This study was approved by the Henry Ford Hospital Institutional Review Board. 


\subsection{Patient Classification for AUC and Impact}

All clinical data preceding CMR was reviewed independently by 2 physicians who characterized patient studies as "appropriate", "uncertain", or "inappropriate" as per the 2006 AUC. An initial sample of 25 patients were independently evaluated by both reviewers and then reviewed collectively so as to minimize inter-reviewer variability. Discrepancies were reviewed by an additional staff cardiologist with training in advanced cardiac imaging to finalize characterization per AUC. Test results were subsequently reviewed, as were downstream orders with attention to discontinued or obviated interventions, including medications, other testing, or surgical/percutaneous procedures. A study was deemed to have had an impact also if it changed or clarified a diagnosis, thus directly influencing downstream decision-making.

\subsection{Statistical Analysis}

Comparisons of discrete variables were performed using Fisher's exact test due to the presence of sparse data. Data associations for common clinical variables including body mass index, ejection fraction, and age were evaluated using 2 sample t-tests. Statistical significance was interpreted as a p-value of $<0.05$.

\section{Results}

\subsection{Population Characteristics}

Most patients undergoing CMR did so as part of an evaluation for new or known cardiomyopathy including evaluation for myocardial viability $(\mathrm{n}=189,79 \%)$. Other indications included evaluation of cardiac mass $(n=13,5.4 \%)$, suspected myocarditis $(\mathrm{n}=18,7.5 \%)$ and valvular abnormalities $(\mathrm{n}=19,7.9 \%)$. Amongst the cardiomyopathy indications, the most common were suspected sarcoidosis ( $\mathrm{n}=36,15.1 \%$ of all CMRs ordered), evaluation for arrhythmogenic right ventricular dysplasia $(\mathrm{n}=28,11.7 \%)$, myocardial viability testing $(\mathrm{n}=27,11.2 \%)$, hypertrophic cardiomyopathy ( $\mathrm{n}=25,10.4 \%$ ), and suspected infiltrative cardiomyopathy $(\mathrm{n}=16,6.7 \%)$. The leading indications for CMR at our institution are shown in Table 1.

Table 2 outlines the patient characteristics of those who underwent CMR in 2011 and thus were included in our analysis.

There was no statistically significant difference in appropriateness of the study

Table 1. Leading 5 indications for cardiac magnetic resonance imaging (CMR).

\begin{tabular}{cc}
\hline Indication for CMR & $\mathrm{N}=239$ \\
\hline Cardiomyopathy & $141(59.0 \%)$ \\
Viability, Chest Pain, Myocardial Infarction or ICD Evaluation & $48(20.1 \%)$ \\
Cardiac Mass & $13(5.4 \%)$ \\
Valve Pathology & $18(7.5 \%)$ \\
Myocarditis & $19(7.9 \%)$ \\
\hline
\end{tabular}


Table 2. Patient demographics.

\begin{tabular}{cc}
\hline Patient Demographics & $\mathrm{N}=239$ \\
Female & $101(42.3 \%)$ \\
Tobacco & $62(25.9 \%)$ \\
Hypertension & $104(43.5 \%)$ \\
Diabetes mellitus & $45(18.8 \%)$ \\
Coronary artery disease & $44(18.4 \%)$ \\
History of prior myocardial infarction & $35(14.6 \%)$ \\
Age (average) & $51.5 \mathrm{years}$ \\
Body mass index (average) & $30.1 \mathrm{~kg} / \mathrm{m}^{2}$ \\
Ejection fraction (average) & $50.9 \%$ \\
\hline
\end{tabular}

ordered by indication for CMR (cardiomyopathy, cardiac mass, myocarditis evaluation, or valve pathology). Likewise, there were no differences in appropriateness of examinations ordered based on patient gender, ejection fraction, known coronary artery disease/myocardial infarction or other traditional coronary artery disease risk factors.

\subsection{Impact and AUC}

Of all CMR studies ordered in the study period, 96\% (229/239) were considered appropriate according to the 2006 AUC. Only $1 \%$ of the studies were characterized as uncertain and 3\% as inappropriate. Appropriate studies were more likely to be associated with changes in management $(90 \%, 206 / 229)$ than studies classified as uncertain or inappropriate $(40 \%, 4 / 10)$ with an odds ratio of 21.5:1 (Table 3).

Some differences regarding impact were observed between studies ordered based on indication for testing. Patients receiving CMR for evaluation of a new or known cardiomyopathy were more likely to have cardiac medications added to their treatment regimen $(\mathrm{p}=0.036)$ and to have interventions obviated $(\mathrm{p}=$ 0.041) relative to patients undergoing evaluation of cardiac mass, possible myocarditis, or valvular pathology (Table 4). As would be expected, completed examinations were more likely to lead to change in management than incomplete studies $(92.5 \%$ vs $0 \%, \mathrm{p}<0.001)$.

Of the 23 appropriate CMRs that did not impact management, 11 (48\%) were incomplete studies (e.g., the patient was claustrophobic or was unable to sustain breath hold as part of study protocol). When incomplete examinations are excluded from statistical analysis, $94.5 \%$ of completed appropriate CMRs impacted downstream management (206/218). Other reasons for an appropriate CMR not impacting management included instances in which a physician chose not to incorporate the CMR result in clinical decision-making (i.e. CMR ordered by a cardiothoracic surgeon for viability showed viable myocardium, but the surgeon nonetheless deferred bypass), as well as studies ordered for known hypertrophic cardiomyopathy (diagnosis and management plan already established based on 
Table 3. Association of management impact and study appropriateness.

\begin{tabular}{ccccc}
\hline Variable & $\begin{array}{c}\text { Appropriate } \\
(\mathrm{N}=229)\end{array}$ & $\begin{array}{c}\text { Inappropriate } \\
(\mathrm{N}=3)\end{array}$ & $\begin{array}{c}\text { Uncertain } \\
(\mathrm{N}=7)\end{array}$ & P-value \\
\hline Management changed & $206(90.0 \%)$ & $0(0.0 \%)$ & $4(57.1 \%)$ & $<0.001(\mathrm{~F})^{*}$ \\
Added medications & $37(16.2 \%)$ & $0(0.0 \%)$ & $0(0.0 \%)$ & $0.759(\mathrm{~F})$ \\
$\begin{array}{c}\text { Discontinued } \\
\text { medications }\end{array}$ & $31(13.5 \%)$ & $0(0.0 \%)$ & $1(14.3 \%)$ & $1.000(\mathrm{~F})$ \\
Led to intervention & $59(25.8 \%)$ & $0(0.0 \%)$ & $1(14.3 \%)$ & $0.599(\mathrm{~F})$ \\
Obviated intervention & $50(21.8 \%)$ & $0(0.0 \%)$ & $0(0.0 \%)$ & $0.423(\mathrm{~F})$ \\
Additional testing & $60(26.2 \%)$ & $0(0.0 \%)$ & $0(0.0 \%)$ & $0.267(\mathrm{~F})$ \\
Canceled testing & $44(19.2 \%)$ & $0(0.0 \%)$ & $1(14.3 \%)$ & $1.000(\mathrm{~F})$ \\
\hline
\end{tabular}

F, Fisher's exact test.

Table 4. Association of management impact and study indication.

\begin{tabular}{cccccc}
\hline Variable & $\begin{array}{c}\text { Cardiomyopathy } \\
(\mathrm{N}=190)\end{array}$ & $\begin{array}{c}\text { Masses } \\
(\mathrm{N}=13)\end{array}$ & $\begin{array}{c}\text { Myocarditis } \\
(\mathrm{N}=18)\end{array}$ & $\begin{array}{c}\text { Valves } \\
(\mathrm{N}=19)\end{array}$ & P-value \\
\hline Management changed & $167(87.9 \%)$ & $11(84.6 \%)$ & $16(88.9 \%)$ & $17(89.5 \%)$ & $0.971(\mathrm{~F})$ \\
Added medications & $28(14.7 \%)$ & $1(7.7 \%)$ & $7(38.9 \%)$ & $1(5.3 \%)$ & $0.036(\mathrm{~F})^{*}$ \\
Discontinued medications & $25(13.2 \%)$ & $4(30.8 \%)$ & $3(16.7 \%)$ & $0(0.0 \%)$ & $0.065(\mathrm{~F})$ \\
Led to intervention & $49(25.8 \%)$ & $2(15.4 \%)$ & $2(11.1 \%)$ & $7(36.8 \%)$ & $0.283(\mathrm{~F})$ \\
Obviated intervention & $46(24.2 \%)$ & $2(15.4 \%)$ & $0(0.0 \%)$ & $2(10.5 \%)$ & $0.041(\mathrm{~F})^{*}$ \\
Additional testing & $46(24.2 \%)$ & $3(23.1 \%)$ & $7(38.9 \%)$ & $4(21.1 \%)$ & $0.580(\mathrm{~F})$ \\
Canceled testing & $39(20.5 \%)$ & $2(15.4 \%)$ & $2(11.1 \%)$ & $2(10.5 \%)$ & $0.698(\mathrm{~F})$ \\
\hline
\end{tabular}

F, Fisher's exact test.

other parts of the evaluation).

\section{Discussion}

In today's medical practice environment, the overall rising cost of healthcare has led to an increased awareness of resource utilization, specifically with regard to advanced imaging modalities. In an effort to reduce cost, professional societies have developed appropriateness criteria to assist providers' decision-making when ordering these advanced studies. While these criteria are increasingly referenced by providers and third-party payers alike, data is sparse to validate recommendations that were formed based on expert opinion [1] [3] [4] [5]. While some data does exist serving to validate indications for stress CMR, [2] to the best of our knowledge this is the first study to systematically assess the downstream impact of non-stress CMR, based on the AUC, on management and utilization. Our study demonstrates that CMR studies characterized as appropriate by AUC were significantly more likely to impact downstream management. It additionally suggests that when CMR is ordered for evaluation of cardiomyopathy, it may have particular value in either leading to the addition of medical management, or obviating a potential intervention. Of studies characterized as 
appropriate that did not change patient management, the most frequent reason was an incomplete study, with other reasons including physician discretion as well as specific circumstances in patients with known hypertrophic obstructive cardiomyopathy.

The following brief examples from our cohort may serve to better illustrate how CMR may or may not impact management:

Case 1. A 57 year old male who presented to the emergency room with acute shortness of breath, was found with inferior q waves on electrocardiogram, intermediate troponin elevation, depressed LV systolic function with ejection fraction of $30 \%$, and 3 -vessel obstructive coronary disease, not involving the proximal LAD or left main coronary artery, on coronary angiogram, with a chronically occluded right coronary artery. CMR was appropriate in this situation (Indication \#32, “To determine the viability prior to revascularization; to establish likelihood of recovery of function with revascularization or medical therapy") and showed infarcted non-viable right coronary artery territory with otherwise viable myocardium. It also demonstrated incidental finding of layered thrombus adjacent to inferior wall infarcted myocardium. The patient did not undergo coronary bypass surgery but rather was put on anticoagulation, then had PCI of LAD and circumflex arteries. CMR was repeated 3 months after the above therapies were implemented, and showed resolution of thrombus but also ejection fraction $<35 \%$ thus prompting implantation of primary prevention defibrillator.

Case 2. A 19-year-old male with exertional chest pain, non-sustained ventricular tachycardia on Holter monitoring, and asymmetric septal hypertrophy with septal anterior motion of the mitral valve, consistent with hypertrophic cardiomyopathy, underwent CMR. Though appropriate (Indication \#22, evaluation of specific cardiomyopathies (infiltrative, HCM, cardiotoxic), use of delayed enhancement), this CMR corroborated the HCM diagnosis but did not change management as the patient already met diagnostic parameters for HCM, was already started on medical therapy with good effect, and also already met indication for primary prevention defibrillator. Had the patient been refractory to medical therapy, the CMR may have helped plan further transcatheter septal ablation vs surgical myomectomy intervention, but this was not the case.

Case 3. A 71-year-old female with tuberculosis, pulmonary hypertension, and diastolic heart failure presented with leg swelling and orthopnea, with an echocardiogram revealed a very large mass in the right atrium. Differential included myxoma, thrombus, and tumor invasion. CMR was appropriate (Indication 26, "Evaluation of cardiac mass") and revealed an avascular mass adjacent to the right atrium consistent with thrombus. The patient was started on anticoagulation with subsequent resolution of thrombus.

In summary, our findings serve to support the 2006 AUC classification in that appropriate studies are significantly more likely to influence management and utilization than studies characterized as uncertain or inappropriate.

It is our opinion that CMR, used correctly, is of great utility for the elucida- 
tion of disease mechanisms, for diagnosis clarification in the setting of equivocal imaging studies, and for revascularization decision challenges.

\section{Limitations}

A significant majority of CMRs ordered in our study population were characterized as appropriate $(96 \%)$ with only $10(4 \%)$ studies determined to be uncertain or inappropriate. While this finding demonstrates good adherence at our institution with the AUC, it may limit the generalizability of this study to other institutions where ordering practices may be less optimized. Our study is also limited by the small sample size $(\mathrm{N}=239)$ and its single center design. Finally, evaluation of downstream impact is highly subjective, dependent on the reviewers' medical opinion and limited to what is available in the medical records, which may be incomplete.

\section{Conclusion}

As increased attention is paid to the ordering practices of physicians, with specific regard to advanced imaging studies, further validation of the AUC across multiple centers is needed. Our findings serve as preliminary support affirming the AUC as a useful tool to positively impact downstream utilization in patient care.

\section{Compliance with Ethical Standards}

The authors declare they have no conflicts of interest pertaining to the subject matter in this document. All procedures performed in studies involving human participants were in accordance with the ethical standards of the institutional and/or national research committee and with the 1964 Helsinki declaration and its later amendments or comparable ethical standards. For this type of study formal consent is not required.

\section{References}

[1] Medicare Payment Advisory Commission (MedPAC) (2004) Report to the Congress: Growth in the Volume of Physician Services.

https://www.balch.com/-/media/files/insights/publications/2008/05/report-to-congres s-growth-in-the-volume-of-physici/files/report-to-congress-growth-in-the-volume-of -physici/fileattachment/report-to-congress-growth-in-the-volume-of-physici.pdf

[2] McGraw, S., Mirza, O., Bauml, M.A., Rangarajan, V.S. and Farzaneh-Far, A. (2015) Downstream Clinical Consequences of Stress Cardiovascular Magnetic Resonance Based on Appropriate Use Criteria. Journal of Cardiovascular Magnetic Resonance, 17, 35. https://doi.org/10.1186/s12968-015-0137-x

[3] Ferrari, V.A., Whitman, B., Blankenship, J.C., Budoff, M.J., Costa, M., Weissman, J.N. and Cerqueira, M.D., ACC Imaging Council (2014) Cardiovascular Imaging Paymentand Reimbursement Systems: Understanding the Past and Present in Order to Guide the Future. JACC: Cardiovascular Imaging, 7, 324-332. https://doi.org/10.1016/j.jcmg.2014.01.008

[4] Soman, P. and Kelly, R. (2015) Imaging at the 2014 ACC Legislative Conference: A 
Debrief. JACC: Cardiovascular Imaging, 8, 118-120.

https://doi.org/10.1016/j.jcmg.2014.11.004

[5] Hendel, R.C., Patel, M.R., Kramer, C.M., Poon, M., Hendel, R.C., Carr, J.C., Gerstad, N.A., Gillam, L.D., Hodgson, J.M., Kim, R.J., Kramer, C.M., Lesser, J.R., Martin, E.T., Messer, J.V., Redberg, R.F., Rubin, G.D., Rumsfeld, J.S., Taylor, A.J., Weigold, W.G., Woodard, P.K., Brindis, R.G., Hendel, R.C., Douglas, P.S., Peterson, E.D., Wolk, M.J., Allen, J.M. and Patel, M.R., ACCF/ACR/SCCT/SCMR/ASNC/ NASCI/SCAI/SIR (2006) Appropriateness Criteria for Cardiac Computed Tomography and Cardiac Magnetic Resonance Imaging: A Report of the American College of Cardiology Foundation Quality Strategic Directions Committee Appropriateness Criteria Working Group, American College of Radiology, Society of Cardiovascular Computed Tomography, Society for Cardiovascular Magnetic Resonance, American Society of Nuclear Cardiology, North American Society for Cardiac Imaging, Society for Cardiovascular Angiography and Interventions, and Society of Interventional Radiology. JACC: Cardiovascular Imaging, 48, 1475-1497.

Submit or recommend next manuscript to SCIRP and we will provide best service for you:

Accepting pre-submission inquiries through Email, Facebook, LinkedIn, Twitter, etc. A wide selection of journals (inclusive of 9 subjects, more than 200 journals) Providing 24-hour high-quality service User-friendly online submission system Fair and swift peer-review system Efficient typesetting and proofreading procedure Display of the result of downloads and visits, as well as the number of cited articles Maximum dissemination of your research work

Submit your manuscript at: http://papersubmission.scirp.org/ Or contactwjcd@scirp.org 